\title{
Emprego de GPR no estudo de solos e sua correlação com métodos laboratoriais
}

\section{Employment of GPR in the study of soils and its correlation with laboratory methods}

\author{
Everson Cezar ${ }^{*}$; Marcos Rafael Nanni²; \\ Marcelo Luiz Chicati ${ }^{3}$; Roney Berti de Oliveira ${ }^{4}$
}

Resumo

O objetivo deste trabalho foi avaliar algumas propriedades físicas do solo utilizando o georadar associado à equação CRIM, assim como, sua correlação com os métodos laboratoriais. Foram obtidas amostras de solos deformadas e indeformadas em dois transectos, até a profundidade de $0,5 \mathrm{~m}$ para determinação da umidade volumétrica e da porosidade total do solo por métodos laboratoriais. Nestes mesmos locais e profundidades foram feitas investigações com o georadar para determinação da constante dielétrica do solo $(\mathrm{k})$ a qual foi aplicada à equação semi-empírica CRIM para determinação de porosidade total e posteriormente umidade volumétrica. A análise estatística realizada por meio do ("teste t") mostrou a existência de diferença significativa ao nível de $5 \%$ entre os resultados de porosidade total e umidade volumétrica obtidos por métodos laboratoriais e geofísicos, sendo estes, influenciados pela elevada quantidade de argila e de potássio em solução, proveniente da adubação e aplicação de vinhaça. A correlação obtida entre os parâmetros estimados com o GPR e os conseguidos com as análises laboratoriais foi baixa, $(\mathrm{r}=0,58$ para porosidade total e $\mathrm{r}=0,59$ para umidade volumétrica), mostrando a dificuldade em se determinar propriedades físicas por meio de tais técnicas geofísicas.

Palavras-chave: Constante dielétrica, georadar, porosidade total, umidade volumétrica

\begin{abstract}
This work aims to evaluating some physical properties of soil using georadar associated to the CRIM equation, as well as its correlation with laboratory methods. Soil samples were obtained from deformed and undeformed in two transects to a depth of $0.5 \mathrm{~m}$ to determine the soil volumetric humidity and total porosity by laboratorial methods. At the same places and depths, georadar investigations were carried out, to determine the soil dielectric constant (k), which was applied the semi-empirical CRIM equation to determine the total porosity and subsequently volumetric humidity. The statistical analysis, carried out by the ("t test"), showed a significant difference at a level of $5 \%$ between the results for total porosity and volumetric humidity obtained by laboratorial and geophysical methods which are influenced by the high amount of clay and potassium in solution from the fertilizer and vinasse application. The correlation between parameters estimated with GPR and achieved with the laboratory analysis was low $(\mathrm{r}=0.58$ for total porosity and $\mathrm{r}=0.59$ for volumetric humidity), showing the difficulty in determining physical properties through such geophysical techniques.
\end{abstract}

Key words: Dielectric constant, georadar, total porosity, volumetric humidity

\footnotetext{
${ }^{1}$ Eng $^{\circ}$. Agr $^{\circ}$ Doutorando em Agronomia, Universidade Estadual de Maringá, UEM, Programa de Pós-graduação em Agronomia, PGA, Av. Colombo, 5790, CEP 87020-900, Maringá, PR. E-mail: eversoncezar@yahoo.com.br

2 Prof. Dr. PGA, UEM, Maringá, PR. E-mail: mrnanni@uem.br

${ }^{3}$ Prof. Dr. DEC, UEM, Maringá, PR. E-mail: mlchicati@yahoo.com.br

${ }^{4}$ Prof. Dr. CESUMAR, Centro Universitário de Maringá, Maringá, PR. E-mail: roneyberti@yahoo.com.br

*Autor para correspondência
} 


\section{Introdução}

A cana-de-açúcar(Saccharum officinarum) é uma gramínea que possui grande importância econômica para o Brasil, sendo esta uma das principais fontes de divisas para o país (LEMOS FILHO et. al., 2008). No entanto, a produtividade obtida nos canaviais brasileiros encontra-se abaixo do real potencial que a cultura pode oferecer. Assim, faz-se fundamental a pesquisa na área de manejo para que o setor produtivo explore melhor o potencial da planta e do ambiente de produção, diminuindo suas limitações e elevando sua produtividade.

Dentre as limitações a que requer elevado grau de atenção encontra-se relacionada ao solo. O manejo inadequado da camada de interesse leva a degradação do mesmo, prejudicando o desenvolvimento radicular da cana-de-açúcar (CORRÊA et. al., 2001; DURUOHA; BENEZ; CRUSCIOL, 2001). Segundo Medina et al. (2000), as tecnologias de cultivos adotadas para a cultura pelos produtores usineiros são responsáveis por modificações que ocorrem nas propriedades físicas e químicas do solo, alterando ou mesmo comprometendo a sua capacidade produtiva. Essas técnicas empregadas anos após anos provocam modificações no solo que prejudicam o desenvolvimento normal do sistema radicular da planta (CORSINI; MALHEIROS; SACHI, 1986).

A distribuição e penetração das raízes são fundamentais para o estabelecimento e o desenvolvimento da cultura. O crescimento da cana-de-açúcar na parte aérea é o reflexo da sua capacidade em explorar os primeiros centímetros do solo. Eventuais restrições impostas ao sistema radicular causadas por formação de camadas compactas no perfil cultural acarretam em limitações de produtividade devido à falta de aeração no solo e absorção de água e nutrientes.

Desta forma, faz-se necessário o monitoramento dos solos com vista à preservação da sua qualidade para que seja proporcionada produção continua (FIALHO et. al., 2008). A determinação de parâmetros fisicos como a umidade no momento do preparo do solo, plantio e colheita, assim como a porosidade na área durante o desenvolvimento da cultura, são informações importantes, uma vez que indicam qual deverá ser o manejo aplicado para evitar ou diminuir as possiveis restrições ao desenvolvimento radicular. Porém, os métodos atuais para determinação de ambos os parâmetros são destrutivos e trabalhosos, exigindo mão-de-obra treinada, tempo e infraestrutura própria, fugindo à realidade da maioria das usinas brasileiras.

Surge desta forma a necessidade de desenvolver metodologias que sejam mais rápidas e menos onerosas para os estudos investigativos dos solos. Pesquisadores como Hubbart, Grote e Rubin (2002), Grote, Hubbard e Rubin (2003), Galagedara e Parkin (2003), Huisman e Bouten (2003) e Huisman et al. (2003), utilizaram o georadar ou Ground Penetrating Radar (GPR) para estimar a umidade volumétrica do solo.

Paixão, Prado e Diogo (2006) utilizaram o georadar para determinação da umidade volumétrica do solo empregando a equação de Topp, Davis e Annan (1980), a qual estabelece uma relação empirica entre a constante dielétrica $(\mathrm{k})$ e a umidade com base em volume, fundamentada em dados experimentais de laboratório.

Assim como a umidade, a determinação da porosidade do solo por meio do georadar vem sendo estudada por diversos pesquisadores. Greaves et al. (1996), por exemplo, apresentam relações entre as velocidades intervalares e o conteúdo de água em amostras de solo ou rochas permitindo inferir porosidade em solos arenosos.

Além da técnica geofísica, do georadar para determinação de propriedades físicas do solo por meio de reflexão, vários pesquisadores têm utilizado o Method Índex Refractive Complex (CRIM) para determinar a constante dielétrica do solo (WYLLIE; GREGORY, 1953), permitindo assim, fazer inferências sobre as propriedades do mesmo.

De acordo com Junior e Medeiros (2008), o 
modelo CRIM é comumente usado na interpretação de perfis geofísicos de poços para determinar a velocidade das ondas acústicas em meios porosos. Posteriormente, este método foi expandido e adaptado para ondas eletromagnéticas.

Portanto, este trabalho foi desenvolvido com o objetivo de avaliar a porosidade total e umidade volumétrica do solo em área cultivada com cana-deaçúcar utilizando o georadar associado à equação CRIM, assim como, sua correlação com os métodos laboratoriais convencionais.

\section{Material e Métodos}

O trabalho foi desenvolvido em uma área no município de Bom Sucesso, estado do Paraná, delimitada pelas coordenadas geográficas $23^{\circ} 40^{\prime} 35.45^{\prime \prime}$ a $23^{\circ} 40^{\prime} 30.51^{\prime \prime}$ latitude Sul e $51^{\circ} 45^{\prime} 46.62 "$ a $51^{\circ} 45^{\prime} 40.59 "$ longitude Oeste (Figura 1). Sua altitude média é de 580 metros tendo como referência o marégrafo de Imbituba. O solo estudado foi classificado como LATOSSOLO VERMELHO eutroférrico (LVef) textura argilosa/ muito argilosa, segundo Embrapa (2006), apresentando $681 \mathrm{~g} \mathrm{~kg}^{-1}$ de argila no horizonte A e $794 \mathrm{~g} \mathrm{~kg}^{-1}$ no horizonte B.

Figura 1. Mapa de localização da área de estudo contendo os dois transectos.

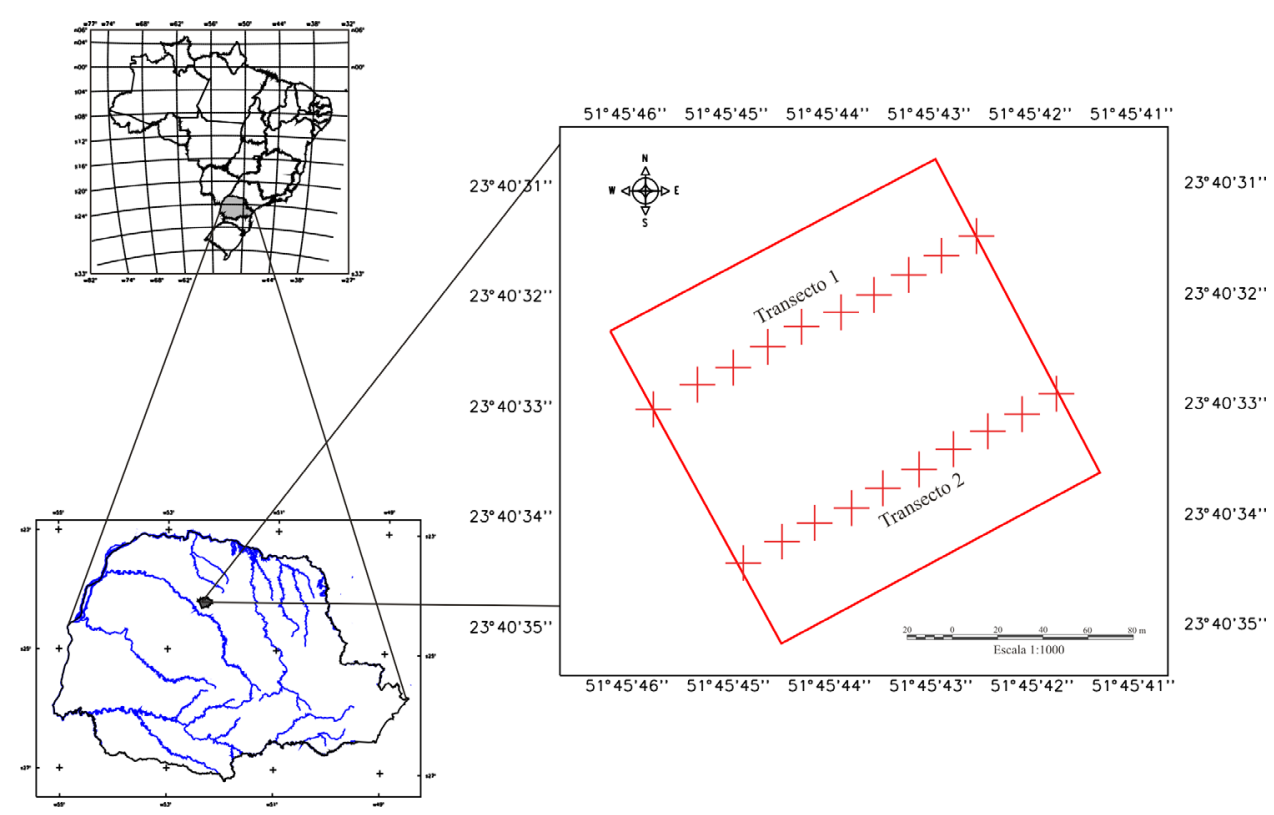

Fonte: Elaboração dos autores.

O clima da região é classificado, segundo Köppen (1948) como Cfa, sendo subtropical úmido mesotérmico com verões quentes e geadas menos freqüentes que outras regiões do estado e tendência de concentração das chuvas nos meses de verão, sem estação de seca definida. A precipitação anual situase em torno de 1500 a $1700 \mathrm{~mm}$ e a temperatura média entre 20 e $22^{\circ} \mathrm{C}$ (CAVIGLIONE et. al., 2000).
Foi delimitada na área de cana-de-açúcar uma parcela experimental com dimensão de 1 ha, onde foram estabelecidos dois transectos, sendo um na entrelinha de plantio, onde o tráfego de máquinas é mais intenso, e outro na linha de cana-de-açúcar, onde não há tráfego. Em cada transecto foram demarcados 10 pontos para coleta das amostras de solo indeformadas, respeitando espaçamento de $10 \mathrm{~m}$ entre pontos. Após terem sido realizadas 
estas amostragens, nestes mesmos pontos foram realizadas amostragens de solo deformadas para determinação da umidade gravimétrica e a investigação com o sistema GPR.

A amostragem indeformada para determinação da densidade e porosidade total do solo foi realizada utilizando-se amostrador de solo e cilindros volumétricos com 5 centímetros de altura e diâmetro, nas profundidades de 0 a $0,1 \mathrm{~m}$, de 0,1 a $0,2 \mathrm{~m}$, de 0,2 a $0,3 \mathrm{~m}$, de 0,3 a $0,4 \mathrm{~m}$ e de 0,4 a 0,5 $\mathrm{m}$. Foram obtidas 50 amostras de solo nas linhas da cultura e 50 nas entrelinhas.

Para determinação da umidade do solo no momento do levantamento de campo realizado com o georadar, foram realizadas amostragens com trado tipo holandês nos mesmos intervalos de profundidade descritos para coleta de amostras indeformadas. As amostras foram acondicionadas em embalagens plásticas e armazenadas em caixas térmicas para evitar perda de água, sendo posteriormente enviadas ao laboratório. A umidade gravimétrica foi determinada segundo Embrapa (1997). Posteriormente, a umidade gravimétrica foi convertida em umidade volumétrica por meio da densidade do solo utilizando-se a equação 1 :

$$
\theta=\mathrm{u} * \mathrm{ds}
$$

onde:

$\theta=$ umidade volumétrica $\left(\mathrm{m}^{3} \mathrm{~m}^{-3}\right) ;$

$\mathrm{u}=$ umidade gravimétrica $\left(\mathrm{kg}_{\text {água }} \mathrm{kg}_{\text {solo }}{ }^{-1}\right)$;

ds $=$ densidade do solo $\left(\mathrm{Mg} \mathrm{m}^{-3}\right)$;

A determinação da porosidade total foi realizada segundo Embrapa (1997) utilizando-se mesa de tensão $(-0,006 \mathrm{MPa})$ para a drenagem das amostras indeformadas. A densidade do solo foi determinada de acordo com Embrapa (1997).

Para obtenção dos dados geofísicos foi utilizado o aparelho GPR Ramac - X3M da empresa Mala GeoScience composto por antena blindada de 500 $\mathrm{MHz}$, unidade de controle e computador portátil para registro dos radargramas.
As aquisições dos dados de campo com o georadar foram realizadas nas mesmas profundidades investigadas com trado holandês e cilindro volumétrico, obedecendo a profundidade de 0 a 0,1 $\mathrm{m}$, de 0,1 a $0,2 \mathrm{~m}$, de 0,2 a $0,3 \mathrm{~m}$, de 0,3 a $0,4 \mathrm{~m}$ e de 0,4 a $0,5 \mathrm{~m}$ para posterior avaliação de correlação com os resultados de umidade e porosidade total do solo, determinados pelo laboratório.

Os dados foram adquiridos com antena blindada, sendo esta deslocada sobre a superfície do terreno, nos mesmos locais onde haviam sido coletadas as amostras indeformadas. Assim, em cada ponto foram realizadas cinco investigações com a antena totalizando as cinco profundidades discutidas anteriormente. Em cada profundidade estudada foi necessário produzir uma reflexão (hipérbole) com o auxílio de um cilindro de aço maciço introduzido no solo, apresentando dimensões de $0,07 \mathrm{~m}$ de diâmetro e $0,1 \mathrm{~m}$ de altura, de forma a indicar, na seção GPR, a velocidade de propagação da onda eletromagnética para posterior cálculo da constante dielétrica $(\mathrm{k})$ pela equação 2 :

$$
\mathrm{v}=\mathrm{c} / \sqrt{\mathrm{k}}
$$

no qual:

$\mathrm{v}=$ velocidade da onda eletromagnética no meio (solo);

$\mathrm{c}=$ velocidade da onda eletromagnética no espaço livre $\left(3,0 \times 10^{8} \mathrm{~m} \mathrm{~s}^{-1}\right)$;

$\mathrm{k}=$ constante dielétrica para o solo.

A utilização do cilindro fez-se necessária uma vez que a falta de variação na constante dielétrica do meio com a profundidade representou interfaces de baixa refletividade, conforme descrito por Prado (2000).

Para obter a maior razão sinal/ruído e a melhoria dos registros durante a aquisição dos perfis GPR em campo, foram utilizados 512 traços. Esta escolha foi baseada em comparações entre registros obtidos com diferentes números de empilhamentos de sinais $(128,256,512$ e 1024 traços) na área experimental, 
assim como realizado por Prado (2000). Esse autor ressalta que o aumento no empilhamento 128 para 1024 traços representa um incremento no tempo de aquisição da ordem de 8 vezes, sendo, portanto, um parâmetro importante a ser considerado na programação dos ensaios quando se pensa na otimização dos mesmos.

O número de amostras coletadas foi de 512, com uma janela de tempo de $76 \mathrm{~ns}$, intervalo de amostragem de $0,05 \mathrm{~m}$ e freqüência de amostragem de $6718 \mathrm{MHz}$. Após a aquisição dos dados brutos, utilizando-se o Ramac Groundvision, estes foram processados por meio do Reflexw versão 4.5.5.

Semelhantemente ao procedimento descrito por Prado (2000), o tratamento dos radargramas consistiu na aplicação do filtro subtract-DC-shift para retirada de ruídos de baixa freqüência. No entanto, nenhum ganho foi aplicado durante o processamento dos dados, em face de não terem sido observadas diferenças na resolução ou profundidade de investigação quando comparadas à seção original obtida com a antena de $500 \mathrm{MHz}$, e a seção resultante da aplicação de ganho, realizada para testes.

Após a determinação da constante dielétrica (k), esta foi aplicada na equação semi-empírica CRIM para estimativa da porosidade total (n). No caso da mistura trifásica formada por grão de argila, ar e água, o modelo resulta na equação 3 (GUÉRGUEN; PALCIAUSSKAS, 1994).

$$
\sqrt{\mathrm{k}}=\mathrm{nS}_{\mathrm{r}} \sqrt{\mathrm{k}_{\mathrm{f}}}+(1-\mathrm{n}) \sqrt{\mathrm{k}_{\mathrm{g}}}+\mathrm{n}\left(1-\mathrm{S}_{\mathrm{r}}\right) \sqrt{\mathrm{k}_{\mathrm{ar}}}
$$

no qual:

$\mathrm{k}=$ constante dielétrica relativa ao solo determinada pela equação 2 ;

$\mathrm{k}_{\mathrm{f}}, \mathrm{k}_{\mathrm{g}} \mathrm{e} \mathrm{k}_{\mathrm{ar}}=$ constantes dielétricas do fluido dos poros (água), do grão mineral e do ar, respectivamente;

$\mathrm{n}=$ porosidade total preenchida por água;

$\mathrm{S}_{\mathrm{r}}=$ saturação da amostra por água (fração de espaço dos poros preenchida com água) determinada pela equação 4:

$$
\mathrm{S}_{\mathrm{r}}=\frac{\theta}{\mathrm{Pt}} \times 100
$$

no qual:

$\theta=$ umidade volumétrica $\left(\mathrm{m}^{3} \mathrm{~m}^{-3}\right)$ obtida pela equação 1 ;

$\mathrm{Pt}=$ porosidade total $\left(\mathrm{m}^{3} \mathrm{~m}^{-3}\right)$ obtida de acordo com Embrapa (1997);

Os valores da constante dielétrica para água $\left(\mathrm{k}_{\mathrm{f}}=81\right)$, constante dielétrica para o ar $\left(\mathrm{k}_{\mathrm{ar}}=1\right) \mathrm{e}$ constante dielétrica do grão mineral $\left(\mathrm{k}_{\mathrm{g}}=5\right)$ foram definidos conforme Davis e Annan (1989).

Em seguida, após a determinação da porosidade total (n), foi possível utilizar este valor para estimar indiretamente a umidade volumétrica por meio da equação 5, proposta por Junior e Medeiros (2008):

$$
\theta=\mathrm{S}_{\mathrm{r}} * \mathrm{Pt}
$$

no qual:

Pt $(n)=$ porosidade total do solo determinada pela equação 3;

$\mathrm{S}_{\mathrm{r}}=$ Saturação da amostra determinada pela equação 4;

Assim, os resultados de Pt e $\theta$ estimados por meio do georadar e do modelo CRIM foram correlacionados com os obtidos em laboratório pelos métodos tradicionais descritos em Embrapa (1997).

A análise estatística aplicada aos resultados estimados e obtidos em laboratório foi realizada por meio do confronto de médias, duas a duas, utilizandose o teste " $t$ " de Student a 5\% de probabilidade. A correlação entre ambos os métodos foi realizada no programa estatístico SAS (SAS, 1999).

\section{Resultados e Discussão}

Os resultados obtidos por meio dos dados do georadar mostraram que o mesmo não obteve resposta satisfatória nas investigações rasas até a profundidade de $0,4 \mathrm{~m}$, seja pela baixa freqüência 
da antena, pela textura muito argilosa do solo ou pela zona rasa de interferência que acabaram interferindo nos dados, tornando-os confusos.

A profundidade que melhor atendeu as investigações foi o intervalo de 0,4 a $0,5 \mathrm{~m}$, sendo esta, portanto, utilizada para a discussão dos dados. Nesta profundidade, as reflexões geradas pelo cilindro de aço no formato de hipérboles mostraramse mais nítidas, possibilitando a determinação das velocidades de propagação das ondas, as quais por meio da equação 1 deram origem as constantes dielétricas do solo (Figura 2).
Os resultados de porosidade total e umidade volumétrica obtidos por meio das análises laboratoriais também foram avaliados no intervalo de profundidade de 0,4 a $0,5 \mathrm{~m}$, para que pudessem ser correlacionados com os resultados obtidos pelo georadar.

A análise estatística realizada por meio do teste " $\mathrm{t}$ " mostrou que houve diferença significativa, em nível de 5\% de probabilidade, entre a média de porosidade total determinada pelo georadar (GPR + CRIM) e o método tradicional (laboratório). O mesmo resultado pôde ser observado para a umidade volumétrica no intervalo de profundidade $0,4-0,5 \mathrm{~m}$ (Tabela 1).

Figura 2. Reflexão produzida pelo cilindro de aço introduzido no solo nas profundidades de 0 a $0,1 \mathrm{~m}$, de 0,1 a $0,2 \mathrm{~m}$, de 0,2 a $0,3 \mathrm{~m}$, de 0,3 a 0,4 m e de 0,4 a $0,5 \mathrm{~m}$, para determinação da velocidade de propagação das ondas eletromagnéticas, as quais, posteriormente, proporcionaram a determinação da constante dielétrica do solo.

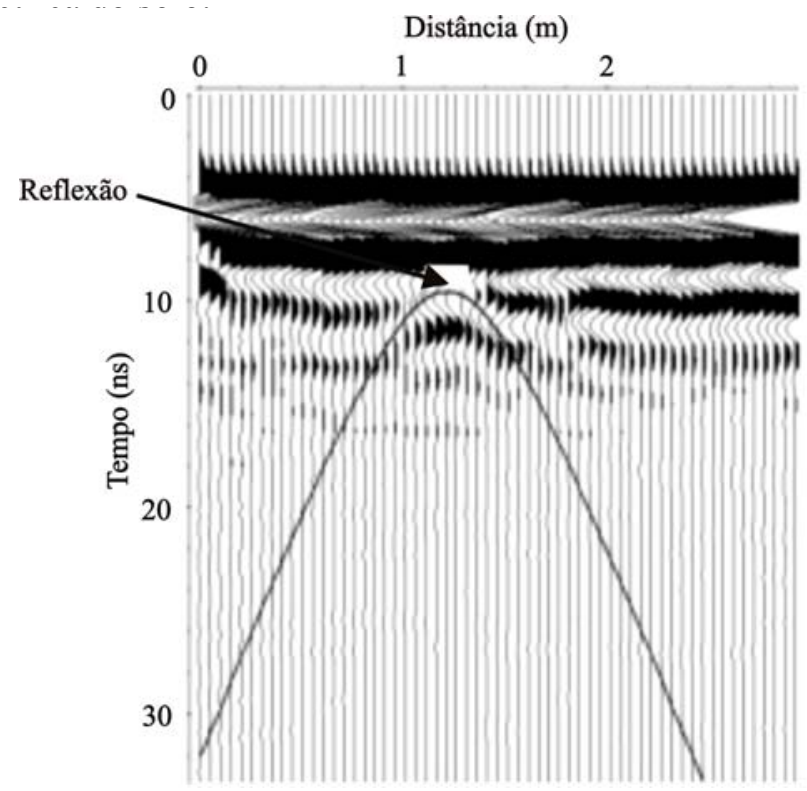

Fonte: Elaboração dos autores.

Tabela 1. Teste " $\mathrm{t}$ " para Pt e $\theta$ obtidas em laboratório e pelo georadar no intervalo de 0,4 a 0,5 m de profundidade.

\begin{tabular}{ccccc}
\hline Variáveis & Profundidade $(\mathrm{m})$ & \multicolumn{2}{c}{ Média \pm Ep } & Valor de $\mathrm{p}$ \\
\cline { 2 - 3 } P. total $\left(\mathrm{m}^{3} \mathrm{~m}^{-3}\right)$ & $0,4 \sim 0,5$ & Laboratório & GPR+CRIM & $0,000135^{*}$ \\
U. volumétrica $\left(\mathrm{m}^{3} \mathrm{~m}^{-3}\right)$ & $0,4 \sim 0,5$ & $0,576 \pm 0,005$ & $0,482 \pm 0,021$ & $0,00259^{*}$ \\
\hline
\end{tabular}

* significativo a $5 \%$ de probabilidade pelo teste "t" de Student.

Ep: erro padrão da média.

Fonte: Elaboração dos autores. 
Esteresultado pode ser explicado pelas limitações encontradas quanto ao uso do equipamento georadar nos trabalhos de campo. Os dados do georadar são muitos sensíveis a presença de sais em solução, e, por tratar-se do quinto ano seguido de cultivo com a cultura da cana-de-açúcar sem renovação da área plantada, a adubação localizada nas linhas associada à aplicação anual de vinhaça, prejudicaram a pesquisa com o GPR.

O potássio presente nos adubos e na vinhaça, a qual, segundo Demattê et al. (2004) é rica neste nutriente pode aumentar a condutividade do meio devido aos íons dissolvidos nos poros, causando assim, forte atenuação das ondas eletromagnéticas, como observado por Pereira et al. (2003). O resultado da análise química na área de estudo mostrou conteúdo elevado de potássio segundo Van Raij et al. (1997), com valor médio de 1,29 $\mathrm{cmol}_{c}$ $\mathrm{dm}^{-3}$ nas linhas e $0,76 \mathrm{cmol}_{\mathrm{c}} \mathrm{dm}^{-3}$ nas entrelinhas de cana-de-açúcar, corroborando assim, a discussão anterior.

Da mesma forma, a grande quantidade de argila encontrada nos horizontes A e B $\left(680,5 \mathrm{~g} \mathrm{~kg}^{-1} \mathrm{e}\right.$ $794,4 \mathrm{~g} \mathrm{~kg}^{-1}$ respectivamente) atenuou a propagação dos campos de ondas eletromagnéticas devido a sua elevada condutividade, reduzindo a profundidade de investigação com esse tipo de equipamento, estando em conformidade com Davis e Annan (1989) e Cezar et al. (2010).

Estas características físicas encontradas na área pesquisada levaram à imprecisão nos radargramas das velocidades de chegada da onda refletida no solo, que são utilizadas para o cálculo da constante dielétrica, gerando valor de constante dielétrica $(\mathrm{k}=3,7)$ não compatível com a realidade da área estudada ( $\mathrm{k}=5$ a 40$)$ como preconizado por Davis e Annan (1989). Assim, a soma destes dois fatores (elevada quantidade de argila e presença de sais em solução principalmente potássio) no decorrer do perfil acabaram influenciando na determinação da constante dielétrica $(\mathrm{k})$.

Portanto, quando $\mathrm{k}$ foi aplicada na equação CRIM, os valores encontrados de porosidade total e, posteriormente, umidade volumétrica, foram mascarados, apresentando estimativa incorreta. Tal fato é confirmado pela baixa correlação existente entre os dados do georadar associados à equação CRIM e aqueles obtidos por meio de análises laboratoriais (Tabela 2 ).

Tabela 2. Correlação entre os dados de porosidade total e umidade volumétrica obtidos em laboratório e pelo georadar no intervalo de 0,4 a $0,5 \mathrm{~m}$ de profundidade.

\begin{tabular}{ccc} 
Variáveis & $\begin{array}{c}\text { P. total observada } \\
\left(\mathrm{m}^{3} \mathrm{~m}^{-3}\right)\end{array}$ & $\begin{array}{c}\mathrm{U} \text { volumétrica observada } \\
\left(\mathrm{m}^{3} \mathrm{~m}^{-3}\right)\end{array}$ \\
\cline { 2 - 3 } $\begin{array}{c}\text { P. total estimada } \\
\left(\mathrm{m}^{3} \mathrm{~m}^{-3}\right)\end{array}$ & $\mathrm{r}=0,58^{* *}$ & - \\
$\begin{array}{c}\text { U. volumétrica estimada } \\
\left(\mathrm{m}^{3} \mathrm{~m}^{-3}\right)\end{array}$ & - & $\mathrm{r}=0,59^{* *}$ \\
\hline
\end{tabular}

** significativo ao nível de $1 \%$ de probabilidade.

- não foram realizadas correlações entre as variáveis.

Fonte: Elaboração dos autores.

Por outro lado, quando se analisa os dados por meio dos métodos tradicionais, não existe influência dos sais ou do teor de argila nos valores encontrados para porosidade total ou umidade volumétrica. Uma vez que as amostras são submetidas à avaliação em laboratório existem metodologias diferenciadas para determinação destes parâmetros (EMBRAPA, 1997), enquanto a medida direta no campo utilizando GPR apresenta limitações pela influência de vários fatores (PRADO, 2000), já que 
a onda eletromagnética se propaga por uma faixa de solo que apresenta propriedades atenuantes como dispersão geométrica da frente de ondas, efeito de polarização (absorção) e dispersões que não podem ser controladas (AGUIAR, 2005).

Esta diferença existente entre as limitações metodológicas ocorridas durante as investigações é o principal fator que explica a baixa correlação para os valores de Pt e $\theta$ determinados pelo georadar e pelos métodos tradicionais, discordando de Causse e Sénéchal (2006).

\section{Conclusões}

O GPR e a equação CRIM associados, não se mostraram capazes de determinar com confiabilidade a porosidade total e umidade volumétrica do solo;

Os resultados de porosidade total e umidade volumétrica obtidos com o GPR diferenciam-se estatisticamente daqueles obtidos em laboratório;

A elevada quantidade de argila no horizonte A e B associada aos sais em solução principalmente o potássio prejudicaram a determinação das propriedades físicas que se pretendia avaliar por meio de técnica geofísica;

A correlação entre as técnicas geofísicas e métodos laboratoriais foi baixa, não sendo possível em tais condições utiliza-las.

\section{Agradecimentos}

À CAPES pela concessão de bolsa de Mestrado ao primeiro autor, ao $\mathrm{CNPq}$ pela bolsa de Produtividade (Proc. n.310948/2006-1) ao segundo autor e bolsa de doutorado (Proc. n.134501/2004-7) ao terceiro autor.

\section{Referências}

AGUIAR, J. C. M. Radar de penetração no solo (GPR): aspectos geofísicos e geodésicos processamento e análise em pavimento flexível. 2005. Dissertação (Mestrado em
Ciências Geodésicas e Tecnologia da Informação) Universidade Federal de Pernambuco, Pernambuco.

CAUSSE, E.; SÉNÉCHAL, P. Model-based automatic dense velocity analysis of GPR field data for the estimation of soil properties. Journal of Geophysics and Engineering, Philadelphia, v. 3, n. 2, p. 169-176, 2006.

CAVIGLIONE, J. H.; KIIHL, L. R. B.; CARAMORI, P. H.; OLIVEIRA, D. IAPAR. 2000. Disponível em: $<$ http:// www.iapar.br/modules/conteudo $>$. Acesso em: $11 \mathrm{dez}$. 2008.

CEZAR, E.; NANNI, M. R.; CHICATI, M. L; FABRIO, F. D.; HATA, F. T.; OLIVEIRA, R. B. Uso de sistema GPR (Ground Penetrating Radar) na avaliação de atributos de um solo sob plantio de cana-de-açúcar. Revista Brasileira de Ciência de Solo, Viçosa, MG, v. 34, n. 2, p. 291-297, 2010

CORREAA, J. B. D.; ANDRADE, L. A.; DIAS JUNIOR, M. de S.; ALVES, V. G. Influência da compactação na concentração de nutrientes da parte aérea da cana-deaçúcar, em três tipos de solos. Revista Stab - Açúcar, Alcool e Subprodutos, Piracicaba, v. 19, n. 5, p. 34-37, 2001.

CORSINI, P. C.; MALHEIROS, E. B.; SACHI, E Sistemas de cultivo da cultura de cana-de-açúcar: efeitos na retenção de água e na porosidade do solo. Revista Brasileira de Ciência do Solo, Campinas, v. 10, n. 1, p. 71-74, 1986

DAVIS, J. L.; ANNAN, A. P. Ground-penetrating radar for high-resolution mapping of soil and rock stratigraphy. Geophysical Prospecting, Netherlands, v. 37, n. 5, p. 531-551, 1989.

DEMATTÊ, J. A. M.; GAMA, M. A. P.; COOPER, M.; ARAÚJO, J. C.; NANNI, M. R.; FIORIO, P. R. Effect of fermentation residue on the spectral reflectance properties of soils. Geoderma, Amsterdan, v. 120, n. 3-4, p. 187-200, 2004.

DURUOHA, C.; BENEZ, S. H.; CRUSCIOL, C. A. C. Desenvolvimento do sistema radicular da cana-de-açúcar (Saccharum spp.) em função da compactação, do tipo de solo e do teor de água. Revista Energia na Agricultura, Botucatu, v. 16, n. 3, p. 35-46, 2001.

EMPRESA BRASILEIRA DE PESQUISA AGROPECUÁRIA - EMBRAPA. CNPS. Manual de métodos de análises de solos. 2. ed. Rio de Janeiro: Embrapa, 1997. 212 p.

Sistema brasileiro de classificação de solos. 2 . ed. Brasília: Embrapa, 2006. 412 p.

FIALHO, J. S.; GOMES, V. F. F.; OLIVEIRA, T. S. de.; SILVA JUNIOR, J. M. T. da. Indicadores da qualidade 
do solo, em sistemas de rotação, na chapada do Apodi CE. Revista Ciência Agronômica, Fortaleza, v. 39, n. 3, p. 353-361, 2008.

GALAGEDARA, L. W.; PARKIN, G. W. An analysis of the GPR direct ground wave method for soil water content measurement. Hydrological Processes, Canadá, v. 17, n. 18, p. 3615-3628, 2003.

GREAVES, R. J.; LESMES, D. P.; LEE, J. M.; TOKSOZ, M. N. Velocity variations and water content estimated from multioffset, ground-penetrating radar. Geophysics, Massachusetts, v. 61, n. 3, p. 683-695, 1996.

GROTE, K.; HUBBARD, S. S.; RUBIN, Y. Field-scale estimation of volumetric water content using ground penetrating radar ground wave techniques. Water Resources Research, United States, v. 39, n. 11, p. 1-14, 2003.

GUÉRGUEN, Y.; PALCIAUSSKAS, V. Introduction to the physics of rocks. New Jersey: Princeton University Press, 1994. 293 p.

HUBBART, S.; GROTE, K.; RUBIN, Y. Mapping the volumetric soil water content of a California vineyard using high-frequency GPR ground wave data. The Leading Edge, San Antonio, v. 21, n. 6, p. 552-559, 2002.

HUISMAN, J. A.; BOUTEN, W. Accuracy and reproducibility of mapping surface soil water content with the ground wave of ground-penetrating radar. Journal of Environmental and Engineering Geophysics, United States, v. 8, n. 2, p. 67-75, 2003.

HUISMAN, J. A.; HUBBARD, S. S.; REDMAN, J. D.; ANNAN, A. P. Measuring Soil water content with ground penetrating radar: a review. Vadose Zone Journal, Madison, v. 2, n. 4, p. 476-491, 2003.

JUNIOR, J. G. O.; MEDEIROS, W. E. Influência do conteúdo de água, granulometria e minerais pesados sobre as reflexões de gpr em corpos arenosos inconsolidados. Revista Brasileira de Geofísica, Rio de Janeiro, v. 26, n. 4, p. 437-449, 2008.

KÖPPEN, W. M. Climatologia: con un studio de los climas de la terra. México: Fondo de Cultura Económica, 1948. $478 \mathrm{p}$.
LEMOS FILHO, L. C. A.; OLIVEIRA, E. L. de; FARIA, M. A. de; BASTOS ANDRADE, L. A. de. Variação espacial da densidade do solo e matéria orgânica em área cultivada com cana-de-açúcar (Saccharum officinarum L.). Revista Ciência Agronômica, Fortaleza, v. 39, n. 2, p. 193-202, 2008.

MEDINA, C. C.; ROBAINA JÚNIOR, R. A. H.; NEVES, C. S. V. J.; BRINHOLI, O. Sistema radicular de cana-deaçúcar submetida a doses de gesso, calcário e vinhaça. Semina: Ciências Agrárias, Londrina, v. 21, n. 1, p. 7175, 2000.

PAIXÃO, M. S. G.; PRADO, L. R.; DIOGO, L. A. Análise do emprego do GPR para estimar o teor de umidade do solo a partir de um estudo na cidade de São Paulo. Revista Brasileira de Geofisica, Rio de Janeiro, v. 24, n. 2, p. 189-198, 2006.

PEREIRA, A. J.; GÂMBOA, L. A. P.; SILVA, M. A. M. da; RODRIGUES, A. R.; COSTA, A. da A utilização do Ground Penetrating Radar (GPR) em estudos de estratigrafia na praia de Iataipuaçú - Maricá (RJ). Revista Brasileira de Geofisica, Rio de Janeiro, v. 21, n. 2, p. 163-172, 2003.

PRADO, R. L. A sísmica de reflexão rasa e o radar de penetração no solo na investigação geológicogeotécnica em ambientes urbanos. Um estudo na cidade de São Paulo-SP, Brasil. 2000. Tese (Doutorado em Geociências) - Universidade Estadual Paulista, Rio Claro.

SAS INSTITUTE. Statistical analysis system institute. Procedure guide for personal computers. Version 5, Cary, 1999.

TOPP, G.C.; DAVIS, J.L.;ANNAN,A.P. Electromagnetic determination of soil water content: measurements in coaxial transmission lines. Water Resources Research, United States, v. 16, n. 3, p. 574-582, 1980.

VAN RAIJ, B.; CATARELLA, H.; QUAGGIO, J. A.; FURLANI, A. M. C. Recomendações de adubação e calagem para o Estado de São Paulo. Campinas: Instituto Agronômico/Fundação IAC, 1997. 285 p. (Boletim técnico, n. 100).

WYLLIE, M. R. J.; GREGORY, A. R. Formation factors of unconsolidated porous media: influence of particle shape and effect of cementation. Petroleum Transactions AIME, Pennsylvania, v. 198, n. 223, p. 103-110, 1953. 
